\title{
ESCOAMENTO DE AR ATRAVÉS DE EMBALAGENS DE POLPA DE FRUTAS EM CAIXAS COMERCIAIS: EFEITOS SOBRE OS PERFIS DE VELOCIDADE EM TÚNEIS DE CONGELAMENTO ${ }^{1}$
}

\author{
Jaime Vilela de RESENDE ${ }^{2, *}$, Lincoln de Camargo NEVES FILHO ${ }^{3}$, Vivaldo SILVEIRA JR. ${ }^{3}$
}

\begin{abstract}
RESUMO
As variações nas velocidades do ar causadas pela resistência ao escoamento em função da quantidade de embalagens de polpa de frutas (100g), acondicionadas em caixas comerciais durante o processo de congelamento, foram avaliadas e as vazões estimadas, com base em um método de tratamento dos dados experimentais. As velocidades foram medidas antes da passagem do ar pelo empilhamento de caixas na câmara. As medições foram analisadas utilizando-se uma rotina de regressão não linear e as vazões determinadas pelo método de integração numérica dos perfis das velocidades ajustadas. O método apresentou estimação $10 \%$ superior à encontrada pelo método tradicional. Mantendo as condições operacionais do ventilador constantes, a vazão para arranjos de sete camadas resultou ser $62 \%$ inferior à de três camadas e, 50,9\% inferior à de cinco camadas. Estes valores foram proporcionais à redução do espaço livre para escoamento do ar.
\end{abstract}

Palavras-chave: alimentos congelados; frutas-indústria; polpa de fruta.

\section{SUMMARY}

AIR FLOW THROUGH FRUIT PULP PACKS CONDITIONED IN COMMERCIAL BOXES: EFFECT OF THE VELOCITY PROFILE DURING BLAST AIR FREEZING. The changes in air velocity, as a function of stacking of fruit pulp packages (100g), offering a resistance to air flow in commercial boxes were evaluated and the air flow rates estimated during blast freezing operations. Velocities were initially measured prior to passing the air through the multi-layer stacks of boxes in the chamber. Processing of the data was accomplished by applying a non-linear regression routine. Air flow rates were determined by numerical integration from adjusted air flow velocity profiles and appeared to be $10 \%$ higher than those obtained by the traditional procedure. Under constant operating conditions of the fan, the results for the 7 layer arrays of boxed products exhibited a $62 \%$ reduction in the flux of chilling air, in relation to the 3-layer arrays, and 50.9\% decrease with respect to the 5-layer array. These values were directly proportional to the void space available for air flow.

Keywords: frozen foods; fuit industries; fruit pulp.

\section{1 - INTRODUÇÃO}

O ar é escolhido como meio de resfriamento em muitas situações, por exemplo, nos túneis de congelamento com corrente forçada. As duas principais considerações para a utilização do ar em sistemas de refrigeração são: o consumo de energia para movê-lo e passá-lo pelo produto e a distribuição espacial da velocidade do ar ao redor do produto [2].

Outra questão importante é a distribuição do ar em relação à geometria da câmara e a uniformidade da trajetória do escoamento. Dentro deste contexto, o controle do escoamento de ar é um ponto crítico na instalação. No congelamento de alimentos, o perfil de velocidade do escoamento determina a eficiência e a homogeneidade dos tratamentos aos quais o produto está sendo submetido. Em equipamentos usados no processamento de alimentos, o escoamento do ar é geralmente turbulento e transiente. Devido à forte variabilidade da velocidade do ar no espaço e no tempo, a sua medida é tida como um grande problema, produzindo resultados não muito confiáveis e indispensáveis na determinação da vazão e cálculos de transferência de calor.

MIRADE, DAUDIN [5]; PEYRIN, KONDJOYAN, DAUDIN [7]; OWER, PANKHURST [6] têm relatado dificuldades relacionadas às medidas da velocidade do ar na planta. Dentre estas estão: (1) O escoamento é instável; (2) A direção do escoamento do ar é fortemente influen- ciada pela presença de objetos, e, nas proximidades do ponto de medida, esta é mais difícil de ser avaliada; (3) A temperatura do ar é em geral, muito diferente daquela utilizada pelo fabricante para calibrar o aparelho que freqüentemente está em torno de $20^{\circ} \mathrm{C}$.

Dadas dificuldades mencionadas acima, este trabalho tem como objetivos: (i) o desenvolvimento de uma metodologia para a medida da velocidade do ar na câmara de congelamento carregada com caixas contendo o produto, buscando eliminar as dificuldades e fontes de erros inerentes ao escoamento e, (ii) a análise do perfil de velocidade e das vazões do ar na câmara de congelamento e a influência da quantidade de produto no interior das caixas sobre a resistência ao fluxo de ar.

\section{2 - MATERIAL E MÉTODOS}

\section{1 - Localização dos pontos de medidas da velocidade do ar na câmara de congelamento}

Foram feitas dez perfurações na porta da câmara horizontal de congelamento, distribuídas em dois grupos de cinco perfurações espaçadas em intervalos de $0,10 \mathrm{~m}$ em alinhamento vertical (Figura 1). Por estas aberturas foi introduzido o sensor para a medição da velocidade do ar, sendo os pontos de medida, fixos para cada ponto da altura da seção variando-se em espaçamentos iguais de $0,025 \mathrm{~m}$ a profundidade com que o sensor é inserido na câmara. O objetivo foi proporcionar uma to-

\footnotetext{
${ }^{1}$ Recebido para publicação em 08/08/01. Aceito para publicação em 03/12/01.

${ }^{2}$ LEST/FEMEC/UFU-Av. João Naves de Avila 2160 - Bloco 1 M - Campus Santa Mônica - CEP 38400-089 Uberlândia/MG - E-mail: jvresende@mecânica.ufu.br ${ }^{3}$ DEA/FEA/UNICAMP - Cx. Postal 6121 - Cid. Universitária Zeferino Vaz - CEP 13083-970 - Campinas/SP

*A quem a correspondência deve ser enviada.
} 
mada de leituras da velocidade do ar em múltiplos pontos ao longo da seção perpendicular ao fluxo de ar anterior à passagem pelo produto.

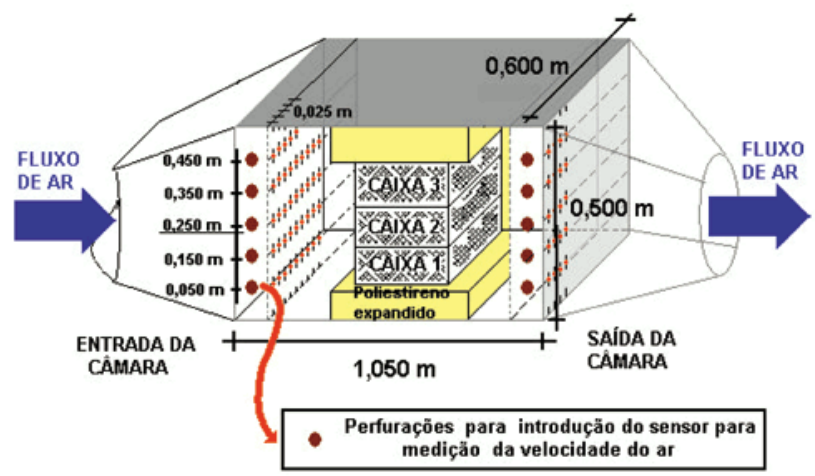

FIGURA 1. Posicionamento dos sensores para leituras da velocidade do ar em múltiplos pontos ao longo da seção da câmara de congelamento.

\section{2 - Procedimento experimental}

Após o estabelecimento das condições operacionais do túnel de congelamento, um empilhamento de três caixas de material plástico contendo as amostras (embalagens de 100 gramas) em arranjos de 7, 5 e 3 camadas, foi colocado na câmara de congelamento. Para evitar a formação de canais preferenciais, placas de poliestireno expandido foram colocadas nas seções laterais das caixas de forma que todo ar passando pela câmara fosse obrigado a passar por entre as caixas contendo o produto. A velocidade do ar foi estabelecida como uma função da quantidade de produto colocado em cada caixa, sendo o empilhamento o obstáculo responsável pela resistência ao escoamento de ar na câmara.

A área disponível $\left(\mathrm{A}_{\mathrm{E}}\right)$ para o escoamento foi avaliada pela seguinte equação:

$A_{E}=A_{\text {Total }}-A_{\text {arranjo }}$

Os valores calculados de $A_{E}, A_{\text {rranjo }}$, e $A_{\text {Total }}$ para experimentos com arranjos de 7, 5 e 3 camadas de produto, bem como as dimensões médias das caixas e das embalagens individuais estão mostradas na Tabela 1 .

TABELA 1. Parâmetros usados na equação (1) para avaliação da área de escoamento.

\begin{tabular}{|c|c|c|c|}
\hline \multicolumn{4}{|c|}{ Dimensões das Amostras (Valores médios) } \\
\hline & Caixas & \multicolumn{2}{|c|}{ Sacos de Polietileno $(100 \mathrm{~g})$} \\
\hline Comprimento $(\mathrm{m})$ & $\mathrm{D} 1=0,585$ & \multicolumn{2}{|c|}{$\mathrm{D} 4=0,1072$} \\
\hline Largura $(\mathrm{m})$ & $\mathrm{D} 2=0,400$ & \multicolumn{2}{|c|}{$\mathrm{D} 5=0,0722$} \\
\hline Altura (m) & $\mathrm{D} 3=0,125$ & \multicolumn{2}{|c|}{$\mathrm{D} 6=0,0160$} \\
\hline \multicolumn{4}{|c|}{ Parâmetros usados na Equação (3.9) } \\
\hline Cm (Camadas) & $\begin{array}{c}A_{\text {Total }}=3 \times \mathrm{D} 1 \times \mathrm{D} 3 \\
\left(\mathrm{~m}^{2)}\right.\end{array}$ & $\begin{array}{c}A_{\text {arranjo }}=5 \times C \mathrm{~m} \times \mathrm{D} 4 \times \mathrm{D} 6 \\
\left(\mathrm{~m}^{2}\right)\end{array}$ & $\begin{array}{l}A_{E} \\
\left(m^{2}\right)\end{array}$ \\
\hline 7 & 0,2194 & 0,1801 & 0,0393 \\
\hline 5 & 0,2194 & 0,1286 & 0,0907 \\
\hline 3 & 0,2194 & 0,0772 & 0,1422 \\
\hline
\end{tabular}

A temperatura do ar foi controlada utilizando resistências elétricas, para uma capacidade frigorífica constante.

Os principais fatores considerados foram temperaturas do ar (3 níveis) e quantidades de produto (3 níveis) e a maneira como foram fixados está mostrada na Tabela 2.

TABELA 2. Fatores e níveis dos fatores considerados na avaliação das medidas da velocidade do ar.

\begin{tabular}{cccc}
\hline Variáveis & \multicolumn{3}{c}{ Níveis } \\
\hline \multirow{2}{*}{ Temperatura do $\operatorname{ar}\left({ }^{\circ} \mathrm{C}\right)$} & $\cong-30$ & 0 & -1 \\
\cline { 2 - 4 } Quant. de produto & 7 Camadas/caixa & 5 Camadas/caixa & 3 Camadas/caixa \\
\hline
\end{tabular}

\section{3 - Medidas da velocidade do ar}

Após o carregamento do produto e com o equipamento em operação, foram feitas as medidas da velocidade do ar utilizando um anemômetro de fio quente (TSI, USA, Modelo.8330-M). As leituras foram pré-estabelecidas em um total de 115 pontos, espaçados ao longo da câmara em triplicata, totalizando 345 leituras para cada corrida. A velocidade do ar foi medida em todos os experimentos num total de 81 ensaios, resultando em 9 repetições para cada tipo de configuração (arranjo de camadas) e temperatura.

\section{4 - Correção das leituras da velocidade do ar para baixas temperaturas}

O instrumento de medição da velocidade do ar usado possui compensação de temperatura, o que significa que, em um escoamento uniforme, as leituras de saída permanecem constantes ao longo de uma faixa de temperatura. O procedimento para a correção da velocidade indicada pelo instrumento $\left(\mathrm{v}_{1}\right)$ para a velocidade real $\left(\mathrm{v}_{2}\right)$ necessária para a compensação da temperatura a partir de dados da temperatura e pressão ambiente foi proposto pelo fabricante (TSI INCOPORATED [8]).

\section{5 - Análises das velocidades do ar na seção da câmara de congelamento}

A análise foi feita, traçando-se o perfil de velocidade do ar na seção da câmara, a partir da média das leituras feitas pelo termoanemômetro após as devidas correções. A partir das curvas de superfície da velocidade do ar em função das coordenadas definidas pela altura (x) e comprimento (y) da câmara obtém-se a superfície de resposta representada pela equação:

$\mathrm{v}=\mathrm{F}(\mathrm{x}, \mathrm{y})$

Os ajustes das superfícies de respostas foram feitos pelos mínimos quadrados utilizando o software TableCurve3D ${ }^{\circledR}$ que faz uma varredura testando várias equações e selecionando a que melhor se ajusta a superfície obtida a partir dos pontos experimentais, com 
opção para a seleção ser feita baseada na melhor correlação $\left(\mathrm{r}^{2}\right)$, desvio padrão ou $\mathrm{F}$ estatístico.

\section{6 - Avaliação da vazão de ar na câmara de congelamento}

A vazão foi avaliada quantificando o volume sob a superfície de resposta das velocidades do ar após o ajuste como descrito anteriormente. Esta avaliação foi feita por integração numérica sob a superfície de resposta pela resolução da equação (3) mostrada a seguir:

$\dot{V}=\int_{a}^{b} \int_{c}^{d} F(x, y) d y d x$

onde $\mathrm{c} \leq \mathrm{x} \leq \mathrm{d}$ compreende o intervalo referente à altura da seção de entrada da câmara de congelamento, sendo igual a $0,050 \leq \mathrm{x} \leq 0,450(\mathrm{~m})$, e $\mathrm{a} \leq \mathrm{y} \leq \mathrm{b}$ refere-se ao comprimento (profundidade) da seção, sendo igual $0,001 \leq \mathrm{y} \leq 0,600(\mathrm{~m})$

A metodologia utilizada é a integração numérica bidimensional pela regra parabólica (ou regra de Simpson bidimensional) cuja resolução se faz através de iterações aplicadas à seguinte equação (4) [3]:

$$
\begin{aligned}
& \int_{a}^{b} \int_{c}^{d} F(x, y) d x d y=\frac{h k}{9}\left[\left(F_{0,0}+4 F_{1,0}+2 F_{2,0}+4 F_{3,0}+2 F_{4,0}+\ldots+F_{m, 0}\right)+\right. \\
& 4\left(F_{0,1}+4 F_{1,1}+2 F_{2,1}+\ldots+F_{m, 1}\right)+2\left(F_{0,2}+4 F_{1,2}+2 F_{2,2}+\ldots+F_{m, 2}\right)+\ldots+ \\
& \left.\left(F_{0, n}+4 F_{1, n}+2 F_{2, n}+\ldots+F_{m, n}\right)\right]+E_{m, n}
\end{aligned}
$$

Para a resolução da equação (4), o intervalo $\mathrm{x}=[0,050$; $0,450]$ foi subdividido em $m=80$ subintervalos de comprimento $\mathrm{h}=(\mathrm{d}-\mathrm{c}) / \mathrm{m}=0,005(\mathrm{~m})$ e $\mathrm{o}$ intervalo $\mathrm{y}=[0,001 ; 0,600]$ em $\mathrm{n}=120$ subintervalos de comprimento $\mathrm{k}=(\mathrm{b}-\mathrm{a}) / \mathrm{n}=0,005(\mathrm{~m})$. Considera-se as funções $\mathrm{F}_{1, \mathrm{j}}=\mathrm{F}\left(\mathrm{x}_{\mathrm{i}}, \mathrm{y}_{\mathrm{j}}\right)(\mathrm{m} / \mathrm{s})$ sendo $\mathrm{y}_{\mathrm{i}}=\mathrm{a}+\mathrm{ike} \mathrm{x}_{\mathrm{j}}=\mathrm{c}+\mathrm{jh}$.

Os valores de $\mathrm{F}\left(\mathrm{x}_{\mathrm{i}}, \mathrm{y}_{\mathrm{j}}\right)$ da velocidade do ar foram gerados pelo mesmo software descrito no item 2.5 e os resultados processados em planilha eletrônica.

\section{3 - RESUlTADOS E DISCUSSÃo}

\section{1 - Correção da velocidade do ar.}

Os valores dos coeficientes para a correção da velocidade do ar para as temperaturas de processamento de $-20,-25$ e $-30{ }^{\circ} \mathrm{C}$ devido à variação da temperatura e umidade relativa do ambiente estão mostrados na Tabela 3. A tabela contém ainda os valores das temperaturas dos pontos de orvalho e pressões de vapor obtidas de cartas psicrométricas.

Em muitos casos o efeito da umidade pode ser desprezivel. Somente com temperaturas e umidades relativamente altas no ambiente, o efeito sobre as leituras pode ser preocupante. Estudos têm mostrado que existem menos que 3\% de mudança na leitura da velocidade do ar quando as variações da umidade relativa do ar na temperatura ambiente são testadas na faixa de $0-100 \%$ [7].
TABELA 3. Fatores para a correção da velocidade do ar indicada pelo instrumento $\left(\mathrm{V}_{1}\right)$ para temperatura, pressão $\left(\mathrm{Fc}_{1}\right)$ e umidade relativa ambiente $\left(\mathrm{Fc}_{2}\right)$.

\begin{tabular}{cccccccc}
\hline & & \multicolumn{3}{c}{$\mathrm{UR}=50 \%$} & \multicolumn{3}{c}{$\mathrm{UR}=80 \%$} \\
\cline { 4 - 8 } Temperatura $\left({ }^{\circ} \mathrm{C}\right)$ & $\mathrm{Fc}_{1}$ & $\mathrm{PO}$ & $\mathrm{P}_{\text {vap }}$ & $\mathrm{F}_{\mathrm{C} 2}$ & $\mathrm{PO}$ & $\mathrm{P}_{\text {vap }}$ & $\mathrm{F}_{\mathrm{C} 2}$ \\
\hline-20 & 0,9176 & $-20,6$ & 0,7404 & 0,9995 & $-20,3$ & 0,7617 & 0,9995 \\
-25 & 0,8994 & $-25,6$ & 0,4570 & 0,9999 & $-25,1$ & 0,4762 & 0,9999 \\
-30 & 0,8813 & $-30,6$ & 0,2737 & 1,0002 & $-30,0$ & 0,2800 & 1,0001 \\
\hline
\end{tabular}

Velocidade real corrigida para a temperatura e pressão, $\mathrm{v}_{2}=\mathrm{Fc}_{1} \mathrm{v}_{1}(\mathrm{~m} / \mathrm{s})$ Velocidade corrigida para umidade relativa do ar, $\mathrm{v}_{3}=\mathrm{Fc}_{2} \mathrm{v}_{2}(\mathrm{~m} / \mathrm{s})$.

$\mathrm{PO}=$ Ponto de orvalho $\left({ }^{\circ} \mathrm{C}\right)$

$\mathrm{P}_{\text {vap }}=$ Pressão de vapor de água do ar ( $\mathrm{mmHg}$ )

O mesmo não se pode dizer em relação aos efeitos da temperatura e pressão do ambiente influenciando diretamente na temperatura de compensação do aparelho. Variações das condições padrões influenciam as leituras produzidas pelo aparelho sendo que os desvios da velocidade indicada em relação à velocidade real são maiores quanto maior for o afastamento das condições do ambiente em relação à condição de referência. Neste trabalho, as condições de temperatura e pressão do ambiente produziram um desvio máximo de - $12 \%$ entre a temperatura indicada pelo anemômetro e a leitura real das velocidades do ar na câmara de congelamento, após as respectivas correções.

\section{2 - Velocidade do ar na câmara de congelamento}

Devido a grande variabilidade da velocidade do ar no espaço e no tempo, para se ter uma representação consistente do perfil de velocidade, torna-se necessária fazer a medida da velocidade em um número de pontos relativamente grande ao longo da seção do escoamento. A variação da velocidade do ar observada nos diferentes tratamentos é devido unicamente à presença das caixas contendo o produto que atuam como obstáculos aumentando a resistência ao fluxo de ar de acordo com a quantidade de produto processada.

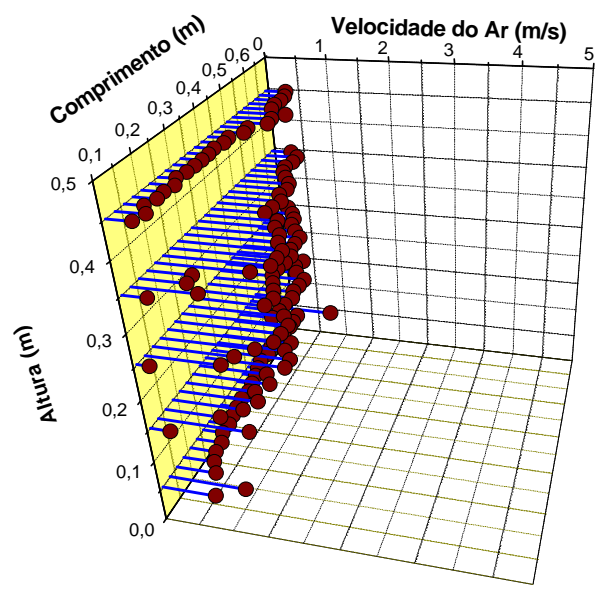

FIGURA 2. Resultados experimentais das velocidades do ar medidas ao longo da seção de entrada da câmara de congelamento para um arranjo de 7 camadas de amostras e temperatura do ar $\left(\mathrm{T}_{\mathrm{ar}}=-25^{\circ} \mathrm{C}\right)$. 
Os gráficos das Figuras 2, 3 e 4 mostram os resultados experimentais das velocidades do ar medidas ao longo da seção da câmara descrevendo a sua variação em função da posição na seção de entrada da câmara de congelamento anterior a passagem pelo produto tendo como coordenadas o comprimento da seção da câmara (y (m)) no eixo das ordenadas e a altura $(\mathrm{x}(\mathrm{m}))$ da seção no eixo das abcissas. As leituras das velocidades são referidas ao eixo $(\mathrm{z}(\mathrm{m} / \mathrm{s}))$ no sistema de coordenadas nos gráficos das figuras. Os gráficos se referem ao processamento de caixas contendo amostras do produto embaladas em sacos de polietileno de 100 gramas dispostos no interior das caixas em arranjos de 7, 5 e 3 camadas respectivamente.

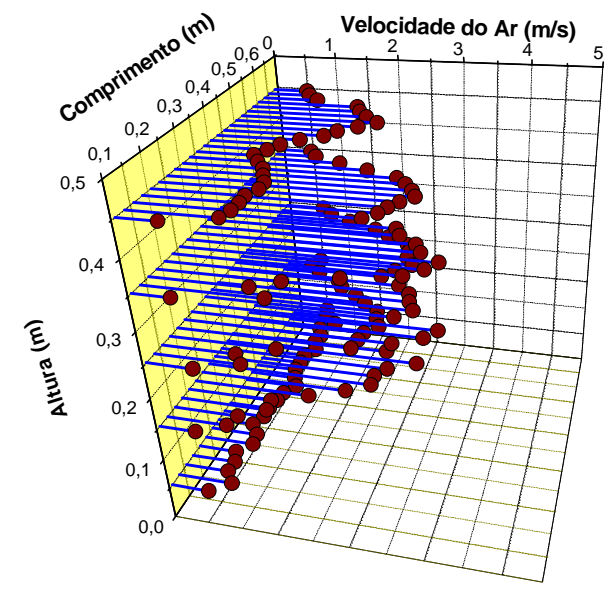

FIGURA 3. Resultados experimentais das velocidades do ar medidas ao longo da seção de entrada da câmara de congelamento para um arranjo de 5 camadas de amostras e temperatura do ar $\left(\mathrm{T}_{\mathrm{ar}}=-25^{\circ} \mathrm{C}\right)$.

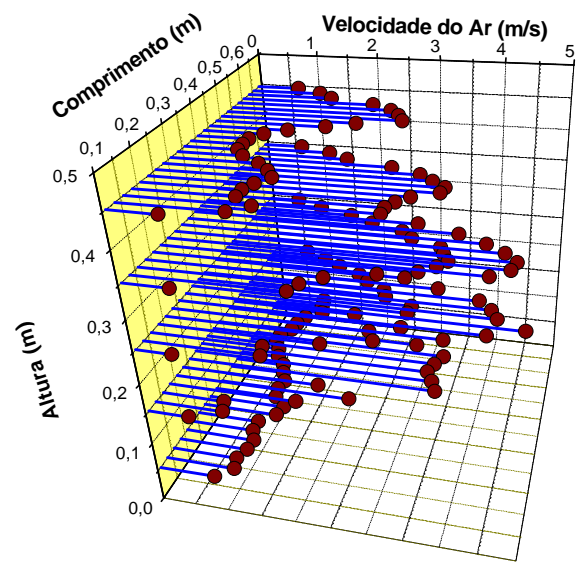

FIGURA 4. Resultados experimentais das velocidades do ar medidas ao longo da seção de entrada da câmara de congelamento para um arranjo de 3 camadas de amostras e temperatura do ar $\left(\mathrm{T}_{\mathrm{ar}}=-25^{\circ} \mathrm{C}\right)$.

A heterogeneidade das medidas obtidas pelo instrumento, pode ser atribuída à mudanças bruscas no direcionamento do escoamento do ar, devido à presença de obstáculos e de acessórios do insuflamento, o que significa que a velocidade do ar varia muito rapidamente em função do tempo e seu valor médio em uma determinada localização se torna de difícil medição. Na tentativa de amenizar estas dificuldades, os resultados experimentais foram ajustados através de regressão não linear, usando um software (TableCurve3D ${ }^{\circledR}$ ) que promove o ajuste, selecionando a equação que produz o melhor coeficiente de correlação ou o menor desvio padrão de acordo com a especificação do usuário. Os gráficos apresentados nas Figuras 5, 6 e 7 mostram a distribuição da velocidade do ar normal à seção de entrada da câmara de congelamento obtida seguindo este procedimento para amostras embaladas em sacos de polietileno e acondicionadas em caixas plásticas em arranjos de 7, 5 e 3 camadas e temperatura do ar de processamento estabelecida a $-25^{\circ} \mathrm{C}$ (nível 2).

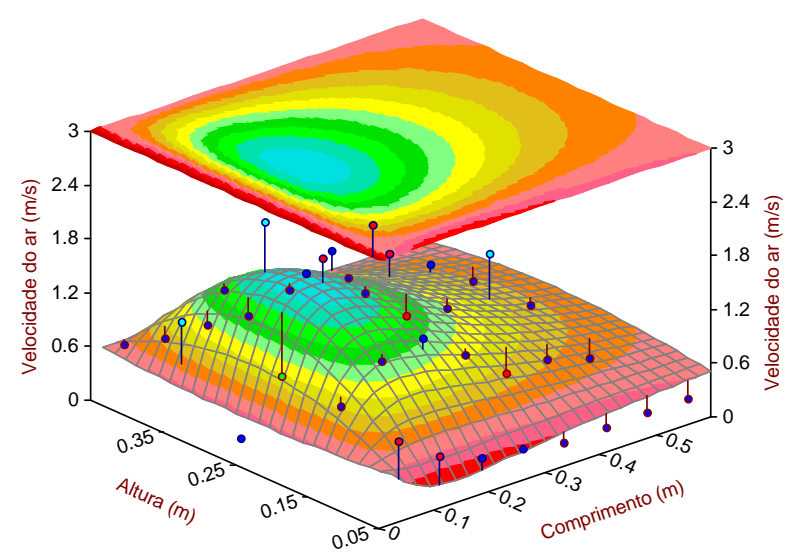

FIGURA 5. Distribuição da velocidade do ar ajustada por regressão não linear normal a seção de entrada da câmara de congelamento para o processamento de amostras contidas em sacos de polietileno no interior de caixas em arranjos de 7 camadas e temperatura do ar $\left(\mathrm{T}_{\mathrm{ar}}=-25^{\circ} \mathrm{C}\right)$.

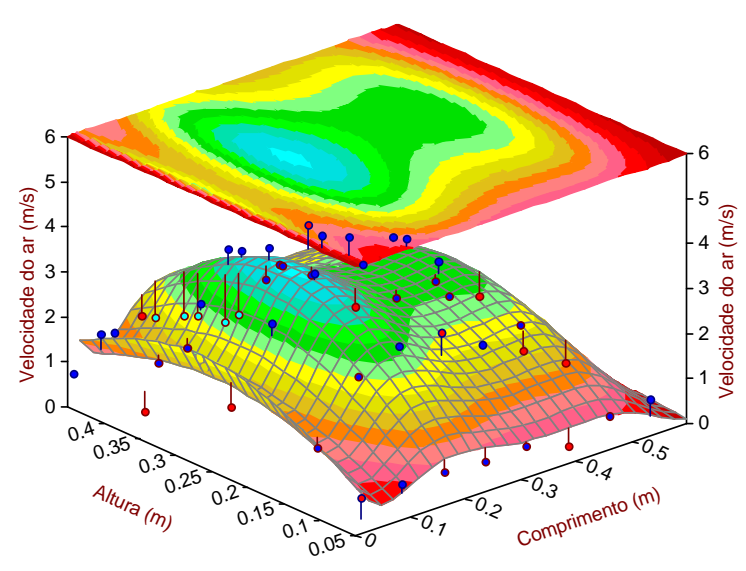

FIGURA 6. Distribuição da velocidade do ar ajustada por regressão não linear na seção de entrada da câmara de congelamento para o processamento de amostras contidas em sacos de polietileno no interior de caixas em arranjos de 5 camadas e temperatura do ar $\left(\mathrm{T}_{\mathrm{ar}}=-25^{\circ} \mathrm{C}\right)$. 
O método de regressão para os ajustes foi a minimização por mínimos quadrados não linear escolhido por proporcionar o melhor coeficiente de correlação $\left(\mathrm{r}^{2}\right)$. O resultado do ajuste é uma curva de resposta superficial para a velocidade em função das coordenadas estabelecidas para a seção de entrada da câmara de congelamento dada pela equação (2), onde $\mathrm{F}(\mathrm{x}, \mathrm{y})$ é a função não linear ajustada. Resultados da análise de variância para os modelos de ajuste das curvas de superfície apresentadas nas Figuras 5, 6 e 7 e os parâmetros necessários para avaliar o grau de correlação entre as respostas observadas e os valores preditos para o modelo ajustado podem ser obtidos, como os apresentados na Tabela 4 para um arranjo de 7 camadas e temperatura do ar de $-25^{\circ} \mathrm{C}$.

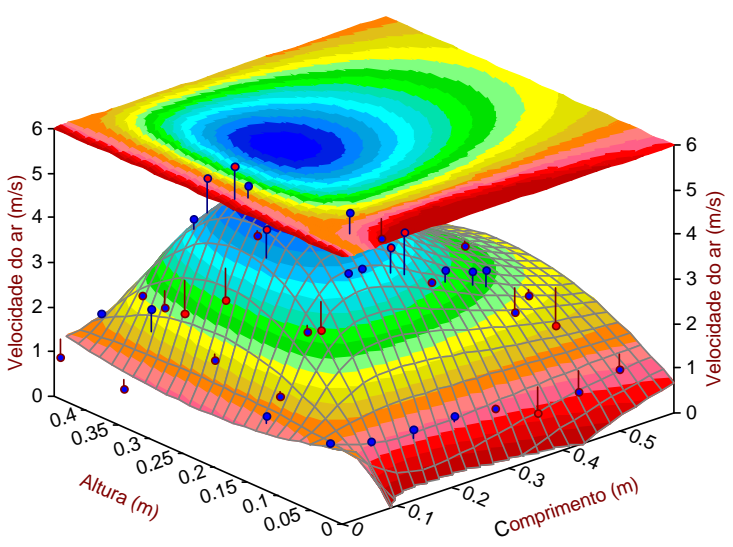

FIGURA 7. Distribuição da velocidade do ar ajustada por regressão não linear na seção de entrada da câmara de congelamento para o processamento de amostras contidas em sacos de polietileno no interior de caixas em arranjos de 3 camadas e temperatura do ar $\left(\mathrm{T}_{\mathrm{ar}}=-25^{\circ} \mathrm{C}\right)$.

TABELA 4. Análise de variância para o modelo ajustado usado na Figura (5) para um arranjo de 7 camadas de amostras embaladas e temperatura do ar de $-25^{\circ} \mathrm{C}$

\begin{tabular}{cccccc}
\hline Fonte & $\begin{array}{c}\text { Soma dos } \\
\text { Quadrados }\end{array}$ & G.L. & M.Quadrática & F-Estat. & P>F \\
& 85,9865 & 7 & 12,2838 & 233,3480 & 0,0000 \\
\hline Regressão & 29,8477 & 567 & 0,0526 & & \\
Resíduo & 115,8357 & 574 & & & \\
Total & 20,0120 & 107 & 0,1870 & 8,7470 & 0,0000 \\
Falta de aj. & 9,8357 & 460 & 0,0214 & & \\
Erro puro & & &
\end{tabular}

Coeficiente de Determinação $\left(\mathrm{r}^{2}\right)=0,74232337351 \mathrm{r}^{2}$ ajustado $=0,7386816678$ Erro padrão do ajuste $=0,229437 \mathrm{r}^{2}$ explicável $=0,9150877072$

O teste usual de significância da equação de regressão ajustada é o teste da hipótese nula que envolve o cálculo do F-estatístico. Comparando-se o valor do Festatístico com o valor tabelado, $\mathrm{F}_{\alpha, \mathrm{p}-1, \mathrm{~N}-\mathrm{p}}$ onde $\mathrm{N}$ é o número de observações, $\mathrm{p}$ o número de parâmetros ajustados e $\alpha$ o nível de significância. Se o valor de F-estatístico excede o valor de $\mathrm{F}_{\alpha, \mathrm{p}-1, \mathrm{~N}-\mathrm{p}}$ tabelado, então infere-se $\operatorname{com} \alpha$ nivel de significância que a variação contabilizada pelo modelo é significativamente maior que a variação não explicada. Em outras palavras, quanto maior o valor de F-estatístico melhor o ajuste. Observa-se que praticamente todos os valores de F-estatístico calculados para os ajustes das curvas mostradas nas Figuras 5, 6 e 7 estão muito acima do valor de $\mathrm{F}$ tabelado indicando que as equações são significativas [4].

Um outro parâmetro apresentado nas tabelas é o coeficiente de determinação $\left(\mathrm{r}^{2}\right)$. $\mathrm{O}$ valor de $\mathrm{r}^{2}$ é uma medida da proporção da variação dos valores da velocidade do ar observada $\left(\mathrm{v}_{\mathrm{u}}\right)$ em torno da média $(\overline{\mathrm{v}})$ explicada pelo modelo ajustado. Nas tabelas de análise de variância, a percentagem de variação explicada pela regressão está por volta de 70 e $85 \%$, mas esse valor não deve ser comparado com $100 \%$ por causa da contribuição devida ao erro puro, que é uma medida do erro aleatório que afeta as respostas [1]- Este deve ser comparado com o valor máximo explicável que percentualmente é dado por $\mathrm{r}^{2}$ explicável nas tabelas acima. Apesar dos valores de F-estatístico nas tabelas indicarem uma regressão altamente significativa, em vários casos existe a evidência da falta de ajuste que se percebe devido ao alto valor de $\mathrm{F}$ específico para a falta de ajuste. A falta de ajuste depende do modelo e será tanto maior quanto mais as estimativas para um dado nível se afastarem do valor médio das respostas determinadas nesse nível.

Uma outra forma de se avaliar a qualidade do ajuste que poderia ser usada como uma segunda razão para a aceitação do modelo, seria a análise dos gráficos dos resíduos. A Figura 8 mostra os gráficos dos resíduos relativos aos experimentos com 7,5 e 3 camadas de produto para os modelos mostrados nas Figuras 5, $6 \mathrm{e}$ 7. Os resíduos são por definição a diferença entre os valores da resposta real observada e aquelas preditas para estas respostas usando o modelo ajustado. Os resíduos deixados pelo modelo devem apresentar uma variância constante e flutuações aleatórias em torno do plano que passa pelo ponto zero, como se pode observar na Figura 8.

Com relação à magnitude dos vetores velocidade que descrevem a distribuição do ar em escoamento, verifica-se visualmente uma diferença considerável quando se observa nos gráficos das Figuras 5, 6 e 7. A variação é causada somente pela quantidade de produto processada que oferece maior ou menor resistência ao escoamento do ar pela câmara. Não se pode afirmar a respeito de se ter uma tendência quanto a estas quantidades, pois os ensaios de 7 camadas possuem uma menor dispersão dos resíduos, porém os ensaios de 3 camadas não possuem a maior dispersão. O que se pode deduzir é que nos arranjos de 7 camadas a não existência de grandes espaços livres para o escoamento obriga o ar a passar entre as embalagens que oferecem resistência ao escoamento em toda a seção da câmara, o perfil na entrada se torna mais uniforme e o gráfico de resíduos para este tipo de arranjo (Figura 7) apresenta uma variância praticamente constante. 


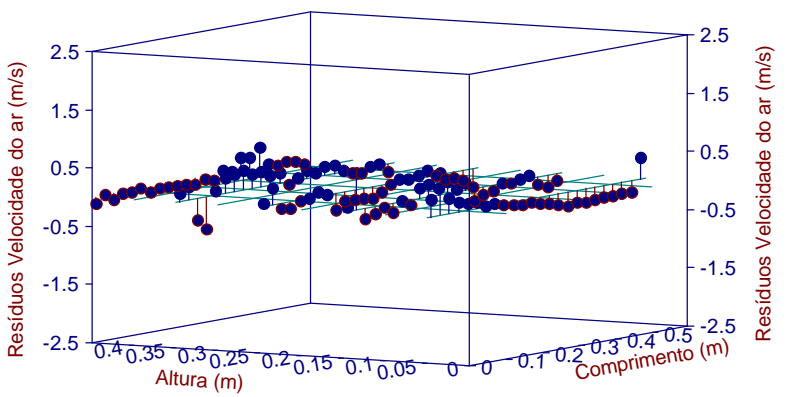

(A)

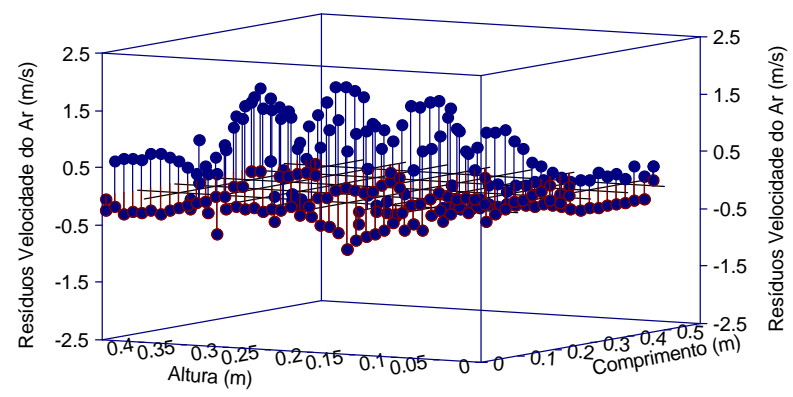

(B)

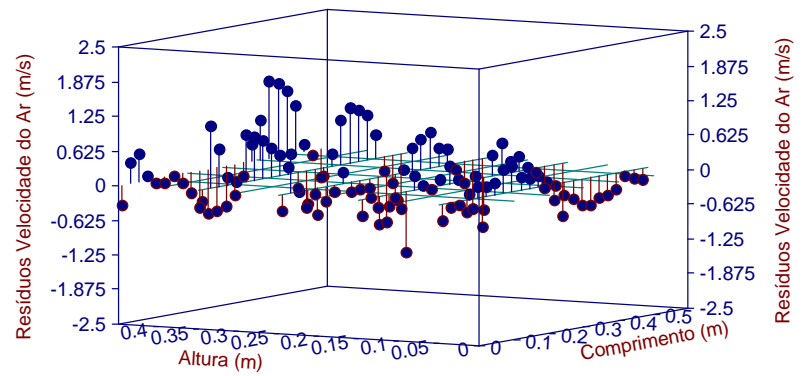

(C)

FIGURA 8. Gráfico de resíduos para o modelo de ajuste da velocidade do ar em experimentos com caixas com arranjos de (A) 7, (B) 5 e (C) 3 camadas de produto.

\subsection{Cálculo da vazão de ar na seção de entrada da câmara de congelamento}

A vazão de ar na câmara de congelamento foi determinada seguindo dois procedimentos: (i) procedimento tradicional que consiste em medir a velocidade local em vários pontos da seção de escoamento e estimar a velocidade média por média aritmética simples, e assim, avaliar a vazão volumétrica pelo produto da velocidade média pela área total da seção de escoamento, e, (ii) método, por integração numérica bidimensional através da determinação da vazão volumétrica sob a superfície de resposta ajustada da velocidade do ar pontual descritas na seção anterior.

A Tabela 5 mostra os valores médios obtidos utilizando as duas metodologias para a determinação das vazões volumétricas durante os experimentos de congelamento de amostras embaladas em sacos de polietileno em caixas com arranjos de 7,5 e 3 camadas e temperaturas do ar em torno de $-20^{\circ} \mathrm{C},-25^{\circ} \mathrm{C}$ e $-30^{\circ} \mathrm{C}$. A última coluna da direita mostra dados comparativos entre os dois métodos avaliados através da diferença percentual com relação a vazão volumétrica determinada pela média integrada entre os resultados obtidos para ambos. A tabela mostra também a quantidade média total de produto processada nas três caixas em cada corrida experimental.

A Tabela 5 mostra em primeiro instante que existe uma diferença considerável entre as duas técnicas de tratamento dos dados de velocidade observados. A técnica utilizada para descrever o escoamento por integração numérica supera os resultados com uma diferença em média de aproximadamente $10 \%$ em relação aos resultados obtidos pelo procedimento tradicional. Uma diferença em torno de $10 \%$ para um parâmetro que descreve este tipo de grandeza pode proporcionar uma seqüência de acúmulo de erros, levando a resultados não conclusivos ao final do processo principalmente quando se refere a cálculos envolvendo transferência de calor, onde, dependendo das condições, se exige um tempo de processamento muito grande. A determinação dos coeficientes de transferência de calor superficiais é extremamente dependente das especificações e regime de fluxos localizados, e as estimativas da carga térmica do produto dependem diretamente da vazão mássica de ar responsável pela retirada de calor do produto durante o processo de congelamento.

TABELA 5. Valores médios da massa total (Kg) de produto processado em diferentes temperaturas e das vazões $\left(\mathrm{m}^{3} / \mathrm{s}\right) \mathrm{de}$ ar na seção da câmara utilizando média experimental e integração numérica

\begin{tabular}{cccccc}
\hline \multicolumn{3}{c}{ Quantidade de Produto } & \multicolumn{3}{c}{ Métodos de determinação da Vazão de ar $\left(\mathrm{m}^{3} / \mathrm{s}\right)$} \\
\hline $\mathrm{T}\left({ }^{\circ} \mathrm{C}\right)$ & Camadas & Massa $(\mathrm{kg})$ & $\begin{array}{c}\text { Média } \\
\text { Experim. }\end{array}$ & $\begin{array}{c}\text { Integ. } \\
\text { Numérica }\end{array}$ & $\begin{array}{c}\text { Dif. relativa } \\
(\%)\end{array}$ \\
\hline \multirow{2}{*}{-20} & 7 & 56,769 & 0,1701 & 0,1914 & 11,14 \\
& 5 & 41,729 & 0,3454 & 0,3941 & 12,35 \\
& 3 & 26,830 & 0,5195 & 0,5959 & 12,82 \\
-25 & 7 & 56,809 & 0,1995 & 0,2220 & 10,15 \\
& 5 & 41,930 & 0,4068 & 0,4564 & 10,85 \\
& 3 & 26,984 & 0,5779 & 0,6520 & 7,41 \\
-30 & 7 & 57,008 & 0,2215 & 0,2137 & $-3,64$ \\
& 5 & 41,719 & 0,3696 & 0,4258 & 13,19 \\
& 3 & 27,157 & 0,4475 & 0,5060 & 11,55 \\
\hline
\end{tabular}

Valores obtidos pela média de 9 repetições.

\section{4 - Efeito da quantidade de produto sobre a vazão de ar na câmara de congelamento}

O diagrama da Figura 9 mostra a redução percentual $(\Delta \mathrm{R} \%)$ da vazão de ar na câmara de congelamento causada pelo aumento da quantidade de produto processada (número de camadas) considerando a mesma rotação do ventilador. O valor de $\Delta \mathrm{R}$ na Figura 9 é calculado pela seguinte equação: 
$\Delta R=\frac{V_{y}-V_{x}}{V_{y}}$

onde $V_{x, y}$ são as vazões de ar avaliadas para as configurações experimentais correspondentes ao número de camadas apresentados como pontos do sistema de coordenadas cartesianas $(\mathrm{x}, \mathrm{y})$ do diagrama.

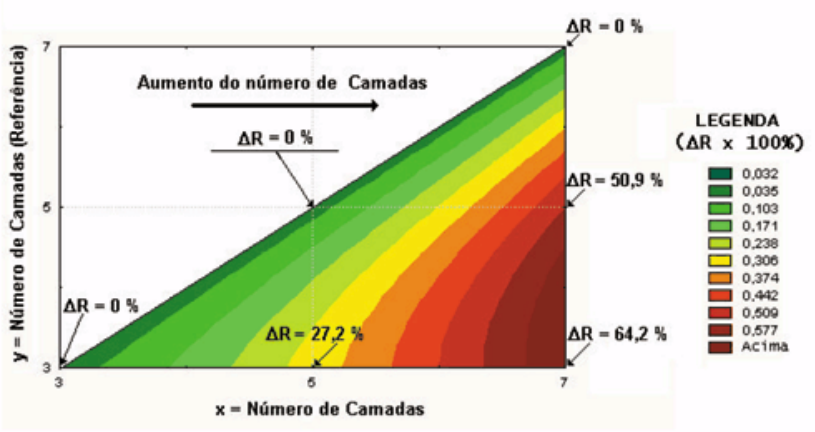

FIGURA 9. Redução percentual da vazão $(\Delta \mathrm{R} \%)$ de ar na câmara de congelamento causada pelo aumento do número de camadas do produto no interior das caixas do empilhamento.

Os maiores valores para as medidas das vazões foram obtidos para experimentos com arranjos de 3 camadas de produto no interior das caixas do empilhamento. Tomando estes valores como referência, observa-se a partir da Figura 9 uma redução de $27,2 \%$ na vazão do ar de resfriamento quando se aumenta de 3 para 5 o número de camadas do produto. Quando o aumento é de 3 para 7 camadas, a redução da vazão do ar de resfriamento passando pelo produto atinge valores em torno de $64 \%$. Se as vazões medidas para os arranjos de 5 camadas são tomadas como referência, a redução da vazão de ar para um aumento de 5 para 7 camadas é cerca de $51 \%$. Estes valores podem ser justificados em termos da redução da área livre para o escoamento do $\operatorname{ar}\left(\Delta \mathrm{A}_{\mathrm{E}} \%\right)$.

A área disponível para o escoamento $\left(\mathrm{A}_{\mathrm{E}}\right)$ é dada pela diferença entre a área total $\left(\mathrm{A}_{\text {Total }}\right)$ da seção normal ao escoamento do ar calculada a partir das dimensões das caixas e da área frontal total dos arranjos $\left(\mathrm{A}_{\text {arranjj }}\right)$ que depende do número de camadas do produto processado no interior das caixas e das dimensões das amostras (sacos de polietileno - 100g) individuais (equação 1).

O gráfico da Figura 10 mostra a correlação entre os valores determinados para a redução percentual da vazão $(\Delta \mathrm{R} \%)$ do ar e a redução da área livre disponível para o escoamento $\left(\Delta \mathrm{A}_{\mathrm{E}} \%\right)$. A redução da vazão causada pelo aumento da quantidade de produto no interior das caixas é diretamente proporcional à redução da área livre para o escoamento do ar.

\section{4 - CONCLUSÕES}

O método de integração numérica da função ajustada da velocidade se apresentou como uma técnica alter- nativa ao procedimento tradicional para a avaliação das medidas das velocidades do ar na câmara de congelamento e para a descrição do fenômeno físico do escoamento do ar. A técnica promove uma varredura dos valores pontuais das medidas da velocidade do ar por toda a seção de escoamento levando em conta a presença de fluxos localizados, permite uma estimativa da vazão por meio de um tratamento matemático e possibilita a detecção e visualização de distúrbios, fenômenos e problemas inerentes do escoamento por métodos estatísticos simples como análise de variância, parâmetros de correlação dos ajustes e análise de resíduos. A técnica utilizada para descrever o escoamento por integração numérica supera os resultados com uma diferença em média de aproximadamente $10 \%$ em relação aos resultados obtidos pelo procedimento tradicional.

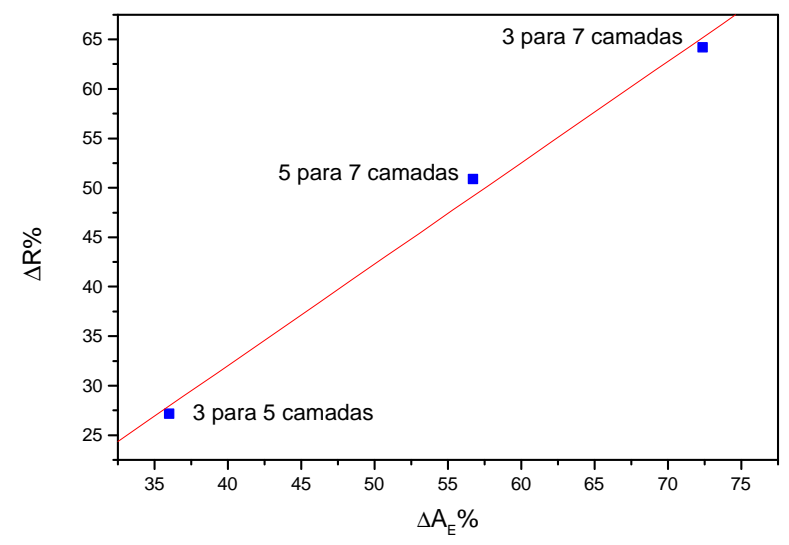

FIGURA 10. Correlação entre a redução da vazão $(\Delta \mathrm{R} \%)$ do ar de resfriamento causada pelo aumento da quantidade de produto nas caixas do empilhamento e a redução da área livre para o escoamento $\left(\Delta \mathrm{A}_{\mathrm{E}} \%\right)$.

O método apresenta como único inconveniente o tempo gasto na obtenção das medidas da velocidade do ar, que poderia ser contornado e realizado com mais informações e maior precisão mediante automação e sensores comunicando a aquisição de dados. Fora o inconveniente, a distribuição espacial dos pontos obtidos é suficiente para análise e detecção de fluxos localizados. Para as mesmas condições operacionais do ventilador, os resultados para arranjos de 7 camadas apresentaram uma redução de $62 \%$ na vazão do ar de resfriamento em relação aos arranjos de 3 camadas e de 50,9\% em relação aos de 5 camadas. Estes dados foram proporcionais à redução da área livre para o escoamento do ar, avaliada pela diferença entre a área total da seção de entrada da câmara e a área especifica para cada tipo de arranjo (3, 5 e 7 camadas).

\section{5 - REFERÊNCIAS BILBLIOGRÁFICAS}

[1] BARROS NETO, B.; SCARMINIO, I. S. e BRUNS, R. E. Planejamento e otimização de experimentos. 2a Edição, Editora da UNICAMP, 1996.

[2] EARLE,R.L. Freezing of foods: An overview Food Engineering and Process Aplications. Proceedings of the Fourth 
International Congress on Engineering and Food, Alberta, Canada, v..2, p. 3-20, 1985.

[3] HOERL, A. E.; NASHED, M.Z.; McKeTTA, J. J. e SILBERBERG, I.H. Mathematics. (eds.) PERRY,H.P. e GREEN,D. Perry's Chemical Engineer's Handbook. Section 2, 6 a edition, McGraw-Hill, Inc., 1984.

[4] KHURI, A. I.; CORNELL, J. A. Response Surfaces - Designs and Analyses. 2nd Edition, Marcel Dekker,Inc., 1996.

[5] MIRADE, P. S.; DAUDIN, J. D. A new experimental methods for visualizing air flow in large food plants, Journal of Food Enginnering, v. 36, p. 31-49, 1998.
[6] OWER,E.; PANKHURST,R.C. The measurement of air flowMéthods of flow measurement based upon rates of cooling of hot bodies. Pergamon Press, England, 1977.

[7] PEYRIN, F.; KONDJOYAN, A.; DAUDIN, J. D. Analyse des dificultés de mesure de la vitesse de l'air dans les industries agro-alimentaires, Industries Agro-Alimentaires et Agricoles, v. 112(10), p. 728-735, 1995.

[8] TSI Incorporated Converting standard velocity to actual velocity. Industrial Test Instruments Application. http://www.tsi.com/iti/homepage/applenote/ti109.htm. 1997b. 